\title{
GEOMETRIA DO RELEVO E ESTRATIGRAFIA DO QUATERNÁRIO COMO BASE À TIPOLOGIA DE CABECEIRAS DE DRENAGEM EM ANFITEATRO - MÉDIO VALE DO RIO PARAÍBA DO SUL
}

\author{
JOSILDA R.S. MOURA*, MARIA N.O. PEIXOTO* e TELMA M. SILVA**
}

\begin{abstract}
RELIEF GEOMETRY AND QUATERNARY STRATIGRAPHY AS A BASIS TO AMPfflTHEATERLJKE HEAD-WATER TYPOLOGY - MIDDLE PARAÍBA DO SUL RIVER VALLEY (BRAZIL). Research on slopes evolution in the Southeastern Brazilian Plateau has demostrated the existence of complex subsurface structures associated to apparently simple topographic features (rampas). It indicates the importance of systematizing stratigraphic studies associated to surface geometry. Considering the amphitheaterlike head-water as a fundamental unit of geomorphological evolution, an amphitheater typology extensive to "drowned" sub-basins is proposed, based on geometric.discontinuities verified at the articulation zone of the side slopes with the longitudinal axis of topographic concavities (hollows). Present knowledge on slope and drainage system dynamics has led to the identification of hollows as segments which are able to reproduce the amphitheater evolution in their subsurface structure. Two fundamental types have been defined. The first is related to the predominance of various episodes of colluviation, convergent to die main axis, defining the structure of rampa complexes in concave hollows (HQ. The second type is related to head-waters which in the recent geologic past have been subjected to intense process of accelerated linear erosion (paleogullies), which is represented in their longitudinal axis by alluvial-colluvial rampas. This is topographically characterized by a subhorizontal to horizontal plane hollow geometry (HCP). The typology proposed may represent an important subsidy in the definition of critical zones to present erosive processes by gullying, surface formations mapping and land-use planning.
\end{abstract}

Keywords: Quaternary, geomorphic evolution, slope and pluvial deposits, head-water, slope geometry.

RESUMO Estudos desenvolvidos sobre a evolução das encostas no Planalto Sudeste do Brasil demonstraram a existência de complexas estruturas de subsuperfície associadas a formas topográficas aparentemente simples (rampas), apontando para a necessidade de sistematizar os estudos estratigráficos acoplados aos de geometria de superfície. Considerando a cabeceira de drenagem em anfiteatro como unidade fundamental de evolução geomorfológica, é proposta uma tipologia de anfiteatros extensiva a sub-bacias de drenagem "afogadas", tendo como base as descontinuidades geométricas verificadas na articulação das encostas laterais com o eixo longitudinal das reentrâncias da topografia (hollows). Os conhecimentos existentes sobre a dinâmica das encostas e dos sistemas de drenagem possibilitaram a identificação do hollow como o segmento capaz de reproduzir em sua estrutura subsuperficial a evolução do anfiteatro. Foram definidos dois tipos fundamentais. O primeiro está relacionado ao predomínio de vários episódios de coluviacão convergentes para o eixo principal, definindo a estrutura dos complexos de rampa em hollows côncavos (HC). O segundo tipo relaciona-se a cabeceiras de drenagem submetidas, no passado geológico recente, a intenso processo de erosão linear acelerada (paleovoçorocas), representado em seu eixo longitudinal por rampas de alúvio-çolúvio, caracterizando a topografia atual por uma geometria de hollow plano, subonzontal a horizontal (HCP). A tipologia proposta pode representar importante subsídio à definição de zonas críticas à retomada erosiva atual, ao mapeamento das formações superficiais e ao planejamento do uso do solo.

Palavras-chaves: Quaternário, evolução geomorfológica, depósitos de encosta e pluviais, cabeceiras de drenagem, geometria de encostas.

INTRODUÇÃO A análise dos principais modelos de evolução de encostas tem evidenciado o caráter dedutivo e limitado dos conhecimentos sobre a atuação dos processos e as formas a eles relacionadas. Do mesmo modo, a diversidade de feições geomórficas e perfis de encosta encontrada nos trópicos úmidos vem ressaltando que as formas de encosta e os processos operantes sobre elas são tão variados e complexos que não é possível atribuir explicações rígidas como as que estabelecem um único modo de desenvolvimento das encostas (Faniran \& Jeje 1983), mesmo considerando-se condições de estrutura, litologia e clima uniformes (Chorley 1964).

As diversas concepções teóricas podem ser resumidas em três correntes principais de estudo da forma das encostas em perfil (Young 1972): a. a que considera a existência de feições básicas, comuns a todas as encostas, independente do substrato, clima ou evolucão morfológica local; b. a que relaciona as formas à atuação diferenciada de variáveis (clima, geologia etc.); c. a que considera as formas como produto do processo histórico de denudação local.

Hack \& Goodlett (1960) introduziram uma perspectiva tridimensional à análise das formas topográficas em cabeceiras de drenagem, estabelecendo classificação para os segmentos de encosta baseada na sua forma geométrica. Segundo esses autores, a área do interflúvio cujos contornos são convexos é definida como nose; a zona aproximadamente retilínea existente entre o segmento convexo e o fundo do vale é denominada side slope; a parte central da cabeceira de drenagem ou qualquer outra área da encosta cujos contornos sejam côncavos é definida como hollow (Fig. 1). Estes diferentes segmentos de encosta apresentam comportamentos hidrológicos distintos, refletindo na distribuição da umidade e da vegetação.

Com o desenvolvimento dos estudos de hidrologia e o progressivo emprego de equações matemáticas na modelagem

\footnotetext{
* Departamento de Geografia, Instituto de Geociências, Universidade Federal do Rio de Janeiro, Cidade Universitária, Ilha do Fundão, CEP 21941, Rio de Janeiro, RJ, Brasil

** Departamento de Geografia, Instituto de Geociências, Universidade do Estado do Rio de Janeiro, Rua São Francisco Xavier, 524, Maracanã, CEP 20550, Rio de Janeiro, RJ, Brasil
} 


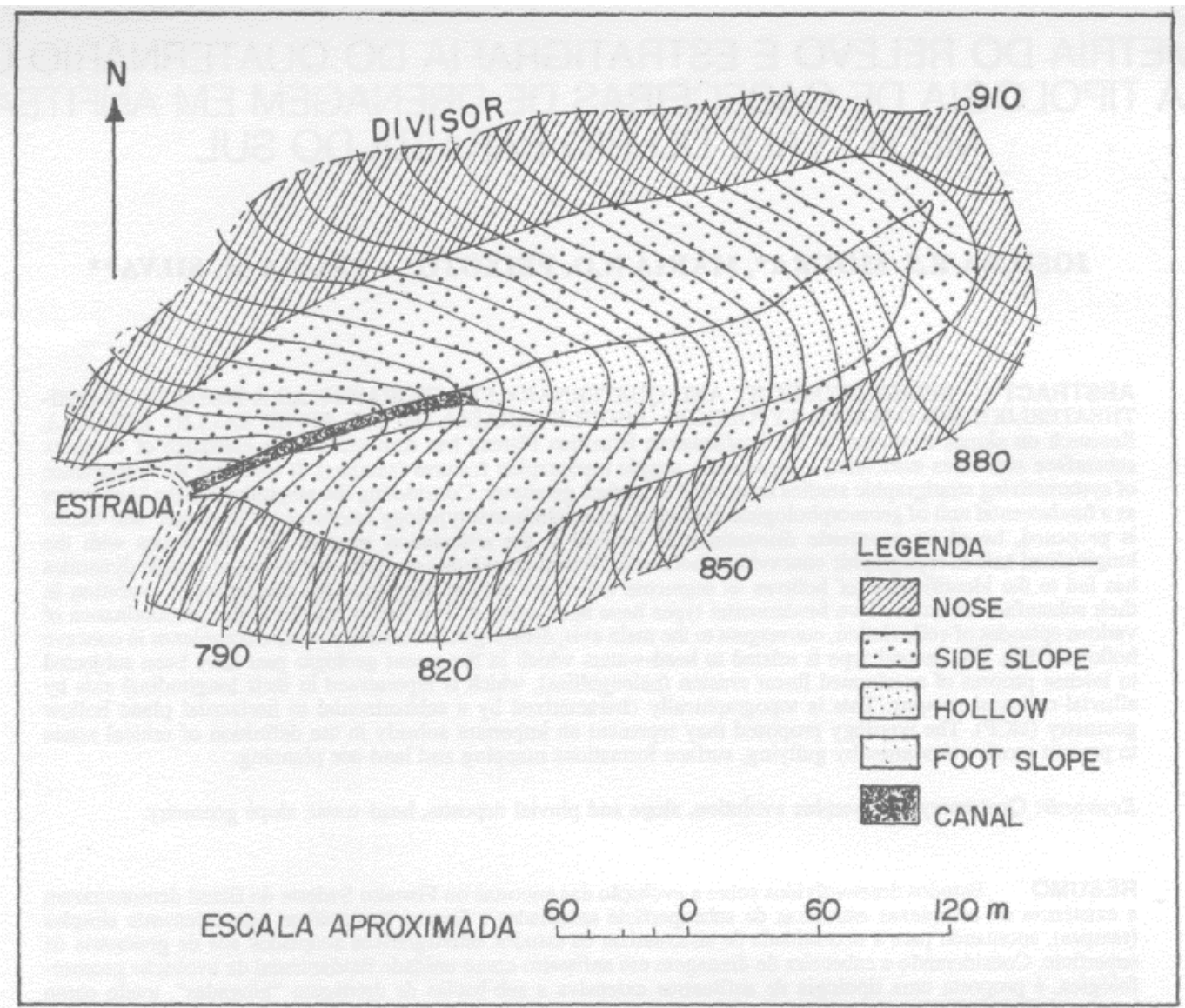

Figura 1 - Topografia de uma cabeceira de drenagem com a representação dos segmentos de encosta propostos por Hack \& Goodlett (1960). A área de contornos côncavos adjacente ao canal é individualizada como foot slope (modificado de Hack \& Goodlett 1960)

Figure 1 - Topographic map of a head-water showing the slope segments proposed by Hack \& Goodlett (1960). The area with concave contours adjacent to the channel is individualized as foot slope (Hack \& Goodlett, modified)

física das encostas, alguns trabalhos buscaram dar enfoque à perspectiva plana ou areal (Troeh 1965), procurando estabelecer os efeitos da curvatura em planta nos processos de encosta. Kirkby \& Chorley (1967) colocam os contornos em planta como uma das quatro variáveis mais importantes para a erosão sob espessas coberturas de solo. Kirkby (1971), aprimorando estas relações, desenvolve suas implicações num modelo de evolução das encostas e, posteriormente, amplia estes conceitos incluindo a atuação dos processos na modificação das formas em planta ao longo do tempo (Carson \& Kirkby 1972).

Ruhe (1975), combinando os três tipos de forma possíveis em planta e perfil (retilínea, côncava e convexa), estabeleceu nove geometrias básicas de encosta dentro da perspectiva tridimensional. Analisando o comportamento hidrológico a partir desta combinação, ressalta que as formas côncavas em planta tendem à concentração dos fluxos e canais de escoamento superficial, podendo gerar voçorocas e canais de primeira ordem.

A abordagem tridimensional dos sistemas de encosta vem subsidiando, nas últimas décadas, a interpretação das relações entre a evolução pedogenética e a do relevo (Bierkland 1974, Gerrard 1981, Ahrnnet 1985, Boardman 1985). Assim, as relações geométricas (planta $\mathrm{x}$ perfil), acopladas à estrutura subsuperficial, individualizam unidades definidas por Hugget (1975) como "Sistemas Solo-Paisagem" (Soil Landscape Systems).

A maioria dos trabalhos produzidos sobre encostas, entretanto, tiveram como base as condições morfogenéticas dos ambientes temperados. A ausência de conhecimentos a respeito da magnitude e freqüência dos eventos e processos geomórficos nos trópicos, o pequeno enfoque dado ao condicionamento recíproco entre formas e processos de encosta, em estudos setorizados, e a utilização de métodos e instrumental técnico inadequados vêm dificultando a apreensão e comparação dos resultados obtidos (Chorley 1964).

Com exceção de alguns trabalhos, como os produzidos por Wigwe (1966), Young (1970, 1972), Swan (1970), Faniran \& Ajaegbu (1971), Ofomata (1973), Jeje (1976 apud Faniran \& Jeje (1983), e mais recentemente Dietrich et al (1986), são muito esparsos os estudos sobre as formas de encosta em áreas tropicais úmidas e subtropicais. Apesar dos anfiteatros ou pequenas bacias de drenagem não-canalizadas (denominadas bacias de zero ordem por Tsukamoto et al 1982) constituírem formas predominantes nas encostas recobertas por espessos regolitos em ambiente tropical e subtropical, pouco se conhece sobre suas características geométricas além da descrição qualitativa como feições que apresentam reentrâncias de contornos côncavos em planta e perfil (Dietrich et al 1986).

Dentro dessa perspectiva, estudos desenvolvidos sobre o Quaternário Superior no Planalto Sudeste do Brasil têm procurado explicar a evolução das encostas com base em argumentos de cunho estratigráfico e geomorfológico (Meis 1977, Meis \& Machado 1978, Meis \& Monteiro 1979, Machado \& Moura 1982, Meis \& Moura 1984, Moura \& Meis 1980, 1986). A articulação da geometria de superfície com a das unidades deposicionais do substrato, numa perspectiva tridimensional, constitui a concepção básica destes estudos. Neste sentido, o termo "complexos de rampa", definido por Meis \& Monteiro (1979), a partir do conceito de "rampa de co- 
luvio", introduzido por Bigarella \& Mousinho (1965), identifica, em abordagem morfoestratigráfica, variações temporais e espaciais no direcionamento dos retrabalhamentos dos materiais de subsuperfície, associadas aos vários episódios de formação de depósitos coluviais dentro das reentrâncias dos anfiteatros (hollows).

O modelo evolutivo proposto por Meis \& Moura (1984) para a região coloca as variações, no nível de base e/ou variações paleoidrológicas, como os fatores responsáveis pela natureza descontínua dos processos de encosta, refletida nos sucessivos retrabalhamentos dos colúvios. Os padrões básicos de comportamento das unidades coluviais observados nos complexos de rampa, associados às feições morfológicas resultantes, levaram à definição de duas condições de evolução de encostas (Meis \& Moura 1984, Fig. 2): a. degradação do nível de base; b. agradação do nível de base. Tais condições são responsáveis, respectivamente, pelo reafeiçoamento total e parcial das encostas, sendo capazes de produzir fenômenos de inversão de relevo.

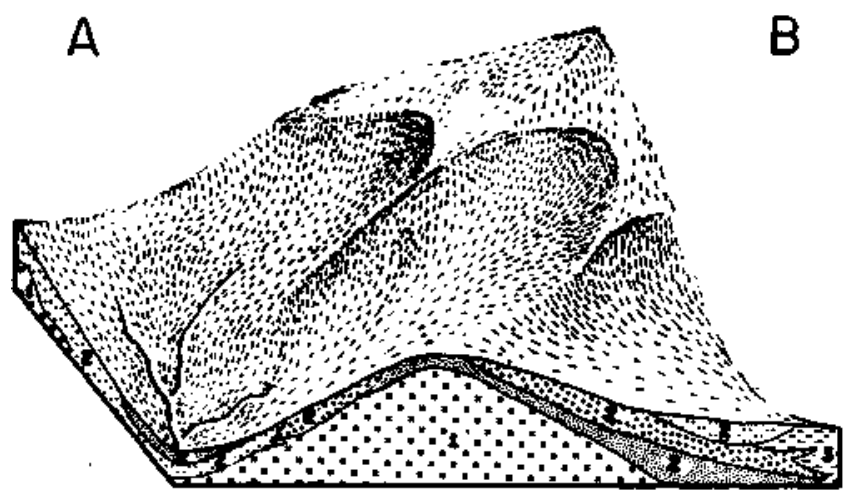

Figura 2 - Bloco diagrama esquemático das condições morfodinâmicas de evolução de encosta definidas por Meis \& Moura (1984): A degradação do nível de base com reafeiçoamento total da topografia das encostas; $\boldsymbol{B}$. degradação do nivel de base com reafeiçoamento parcial das encostas. 1. Embasamento cristalino alterado; 2. depósitos coluviais; 3. depósitos aluviais (Meis et al. 1985)

Figure 2 - Schematc diagram-block $f$ morphodynamic conditions of slope evolution defined by Meis \& Moura (1984): A. base level degradation with total slopes reshaping; B. base level aggradation with partial slopes reshaping; 1. Weathered bedrock; 2. colluvial deposits; 3. alluvial deposits (Meis et al. 1986)

As complexas estruturas de subsuperfície observadas nas encostas convexo-côncavas e nos fundos de vale suavemente inclinados, característicos do domínio de colinas do Planalto SE do Brasil, podem reproduzir, associadas às suas variações geométricas de superfície, padrões evolutivos ligados à dinâmica neoquaternária da paisagem, objeto de estudo deste trabalho.

\section{EVOLUCÃO GEOMORFOLÓGICA E DESCONTINUI- DADES GEOMÉTRICAS E ESTRATIGRÁFICAS NAS CABECEIRAS DE DRENAGEM EM ANFITEATRO A}

proposta de uma tipologia de padrões evolutivos de cabeceiras de drenagem em anfiteatro tem como base a integração de conhecimentos sobre a dinâmica da sedimentação quaternária no Planalto SE do Brasil. A sistematização das investigações, ancorada no modelo de evolução de encostas proposto por Meis \& Moura (1984) e nos estudos de natureza geomorfológica e estratigráfica recentemente desenvolvidos por Moura \& Meis (1986) na região de Bananal (SP/RJ), médio vale do Rio Paraíba do Sul (Fig. 3), permitiu a individualização de domínios morfodinâmicos, caracterizados a partir da análise de sistemas de drenagem diferenciados.

Nessa região, a dinâmica integrada de encostas e fluxos canalizados é documentada na zona de articulação dos segmentos de encosta com os terraços fluviais (rampa-terraço). Tal dinâmica apresenta descontinuidades espaciais e temporais com significado expressivo no Holoceno.

No Pleistocene, a dinâmica de encostas é documentada por uma seqüência de colúvios argilo-arenosos e areno-argilosos de coloração avermelhada, englobados sob a denominação Aloformacão Santa Vitória, em discordância com o embasamento cristalino alterado (gnaisses e migmatitos diversos). A unidade preserva-se nas encostas frontais (head slopes) e íaterais (side slopes). O preenchimento subseqüente dos hollows está relacionado à deposição de materiais coluviais heterométricos de coloração amarelada (Aloformacão Rio do Bananal), que se prolongam localmente pelos fundos de vale; podem preservar um paleorizonte A, datado em 9.800 anos, de grande significado regional. A Aloformacão Cotiara (colúvio arenoargiloso de coloração amarelada) documenta um primeiro reafeiçoamento holocênico da topografia dos anfiteatros. Na seqüência evolutiva, registra-se uma fase de grande instabilidade ambiental, responsável pelo desencadeamento de processos de erosão linear acelerada, promovendo o recuo das encostas a partir de um sistema de voçorocas desenvolvidas tanto nos segmentos côncavos dos anfiteatros quanto no eixo longitudinal das sub-bacias de drenagem menos hierarquizadas. Este mecanismo acarretou aumento no suprimento de material para os leitos fluviais, tendo como conseqüência um entulhamento generalizado dos vales (Foto 1) por sedimentos aluviais predominantemente finos (areno-sílticos e argilosos) - Aloformacão Manso, fácies Campinho - que se interdigitam com materiais alúvio-coluviais e coluviais (Aloformacão Manso, Fácies Quebra-Canto e Fazendinha) relacionados, respectivamente, ao preenchimento dos canais erosivos e retrabalhamento dos materiais de encosta nas cabeceiras de drenagem atingidas pelo fenômeno de erosão linear acelerada holocênica (paleovoçorocas) (Moura \& Mello 1989, Fig. 4).

A dinâmica de entulhamento promoveu a desarticulação dos anfiteatros em relação às calhas fluviais. $\mathrm{O}$ posterior reencaixamento da drenagem no Holoceno (elaboração do terraço superior - TI) e as novas fases de coluviação - Alofarmações Piracema (que engloba materiais castanho-avermelhados, argilo-arenosos e areno-argilosos) e Carrapato (materiais castanhos, areno-argilosos) - condicionaram o reafeiçoamento ou a preservação da topografia originada na fase evolutiva anterior. Concomitantemente, ocorreram fenômenos erosivos acelerados em área (movimentos de massa), documentados por truncamentos nas seqüências deposicionais de encosta.

Esta dinâmica de evolução diferenciada é responsável pela configuração de padrões geomórf icos distintos, mapeáveis em escala de bacias de zero e primeira ordem e sub-bacias de drenagem entulhadas.

O critério geométrico para esta individualização baseia-se nass relações tridimensionais (planta x perfil) entre as encostas laterais e os segmentos que representam as reentrâncias dos anfiteatros (hollows). É de se ressaltar a sensibilidade destes segmentos em reproduzir, em subsuperfície, a natureza dos processos que operaram em sua evolução. As sub-bacias de drenagem afetadas pelo processo de erosão linear acelerada e entulhamento holocênicos reproduzem, em diferentes escalas, a dinâmica de evolução de uma unidade fundamental (anfiteatro).

Considerando a aceitação na literatura geomorfológica e áreas afins da terminologia introduzida por Hack \& Goodlett (1960), ela foi utilizada na tipologia aqui apresentada, e estendida às sub-bacias entulhadas.

O retrabalhamento dos materiais coluviais, convergente para o eixo principal do anfiteatro, relacionado ao desenvolvimento de rampas que se coalescem nas reentrâncias (com- 


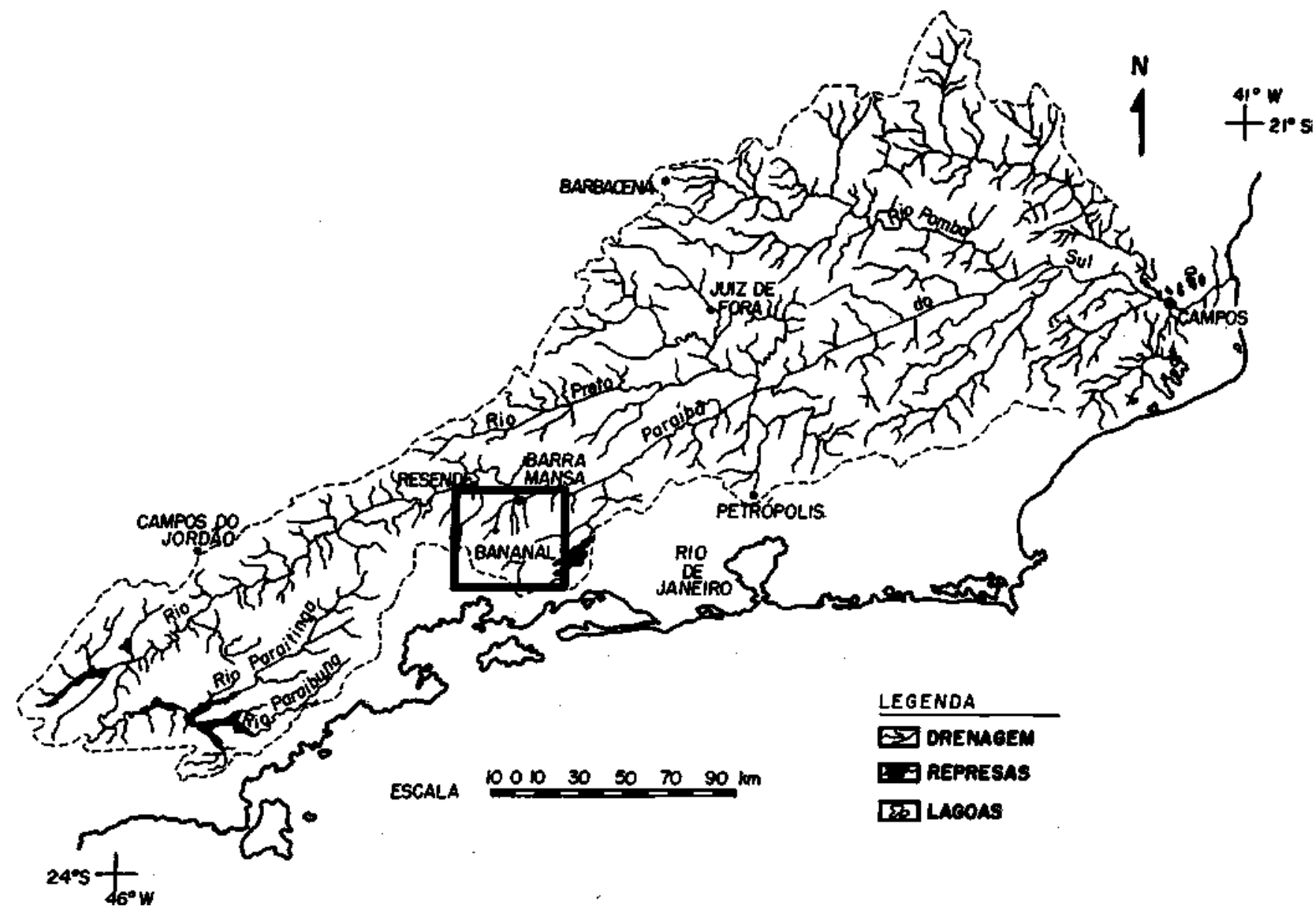

Figura 3 - Mapa da bacia do Rio Paraíba do Sul com a localização da região de Bananal (SP/RJ), área onde têm sido sistematizados os estudos geomorfológicos e estratigráficos voltados à compreensão da evolução da paisagem durante o Quaternário tardio no Planalto SE do Brasil

Figure 3 - Map of Paraíba do Sul River basin with the localization of Bananal region (SP/RJ), where geomorphologic and stratigraphic studies have been developed to improve the understanding of landscape evolution during late Quarternary in middle Paraíba do Sul river valley

plexos de rampa), define a geometria de anfiteatros com hollow côncavo em planta e em perfil (HC). A situação de ruptura brusca das encostas laterais e complexos de rampa, com reentrâncias de fundo plano suborizontal a horizontal, resultante do preenchimento de paleocanais erosivos por materiais alúvio-coluviais (rampas de alúvio-colúvio), define a geometria de anfiteatros e sub-bacias de drenagem entulhadas com hollow côncavo em planta e retilíneo em perfil, denominados anfiteatros com hollow côncavo-plano (HCP) (Foto 2). A estes dois padrões geométricos principais associam-se diferentes estruturas de subsuperfície, cujo registro estratígráfico evidencia variações importantes ao entendimento da história evolutiva (Fig. 5).

De acordo com os registros estratigráficos, os anfiteatros com hollow côncavo apresentam corpos coluviais de geometria inclinada convergentes para o seu eixo longitudinal, evidenciando processos de movimento de massa. Podem conter fases de erosão linear acelerada, documentadas por materiais alúvio-coluviais com geometria de canal, tendo sido as coluviações subseqüentes capazes de efetuar o reafeiçoamento total da paleotopografia (Fig. 6).

Os anfiteatros com hollow côncavo-plano caracterizam-se estatigraficamente pela presença expressiva dos depósitos de natureza alúvio-coluvial preenchendo paleocanais erosivos, que truncam seqüências coluviais mais antigas ou mesmo o embasamento cristalino alterado, estendendo-se desde $p$ vale fluvial até os segmentos de média ou alta encosta (Fig. 7). Estes depósitos geralmente estão recobertos pelas unidades coluviais mais recentes. O recobrimento não chega a descaracterizar a topografia de fundo plano suborizontal a horizontal que identifica os HCP.

Os dois tipos básicos - HC e HCP - foram subdivididos, respectivamente, de acordo com a evolução em relação à drenagem principal e segundo o grau de reafeiçoamento evidenciado pelo ângulo de articulação das encostas laterais com o eixo longitudinal.

Desse modo, os anfiteatros com hollow côncavo (HQ abarcam ainda os subtipos hollow côncavo articulado (HCA), hollow côncavo suspenso (HCS) e hollow côncavo suspenso embutido (HCSe).

Os anfiteatros com hollow côncavo articulado caracterizam-se pela articulação do eixo principal dos complexos de rampa com o nível de base da drenagem atual (Foto 3), documentando que a dinâmica das encostas acompanhou o reencaixamento da drenagem; podem ter evolução relacionada a movimentos de massa ou constituir cabeceiras de drenagem afetadas pelo fenômeno holocênico de erosão linear acelerada, reafeiçoadas pelas coluviações posteriores.

Os anfiteatros com hollow côncavo suspenso encontram-se desarticulados do nível de base atual da drenagem (Foto 4), correspondendo a cabeceiras de drenagem que permaneceram barradas pela sedimentação fluvial/alúvio-coluvial ou que não foram atingidas pelo reencaixamento responsável pelo esvaziamento do vale adjacente, preservando-se, portanto, como cabeceiras desarticuladas. Sua evolução está, em geral, ligada a movimentos de massa. Nas cabeceiras de drenagem que 


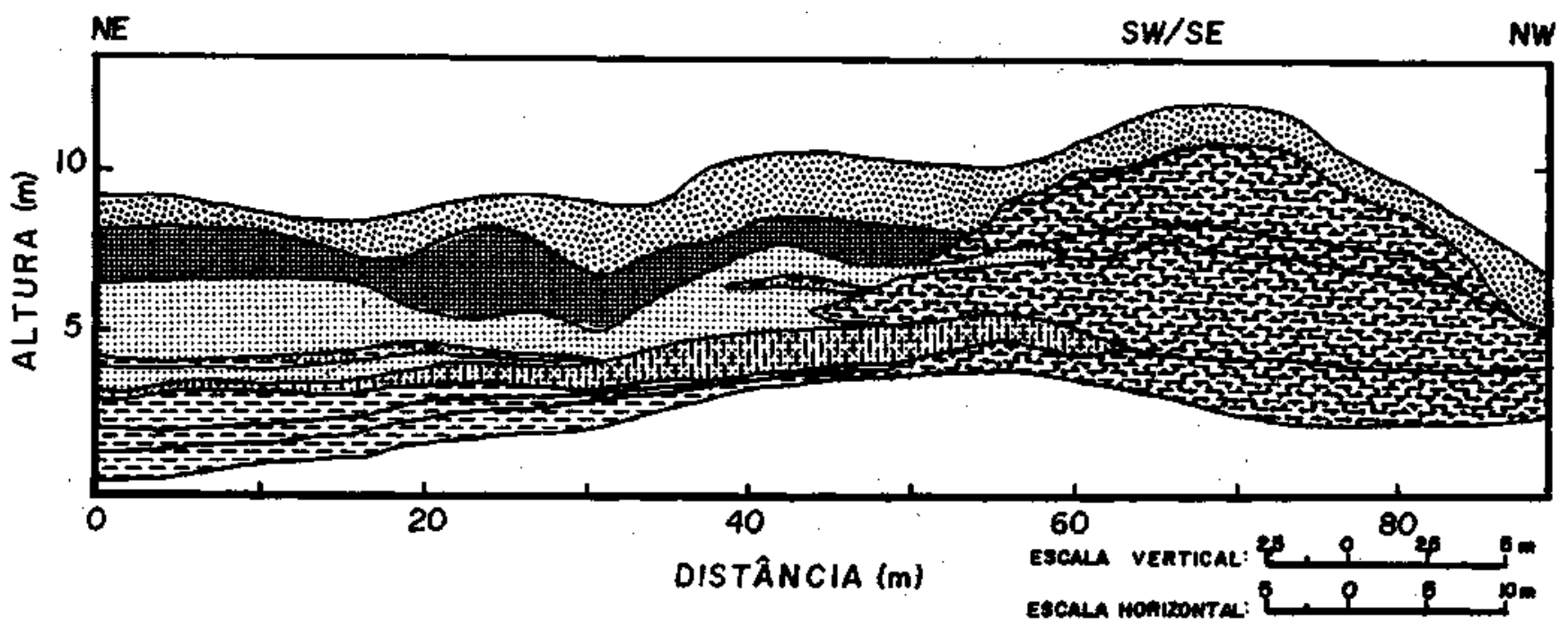

LEGENDA

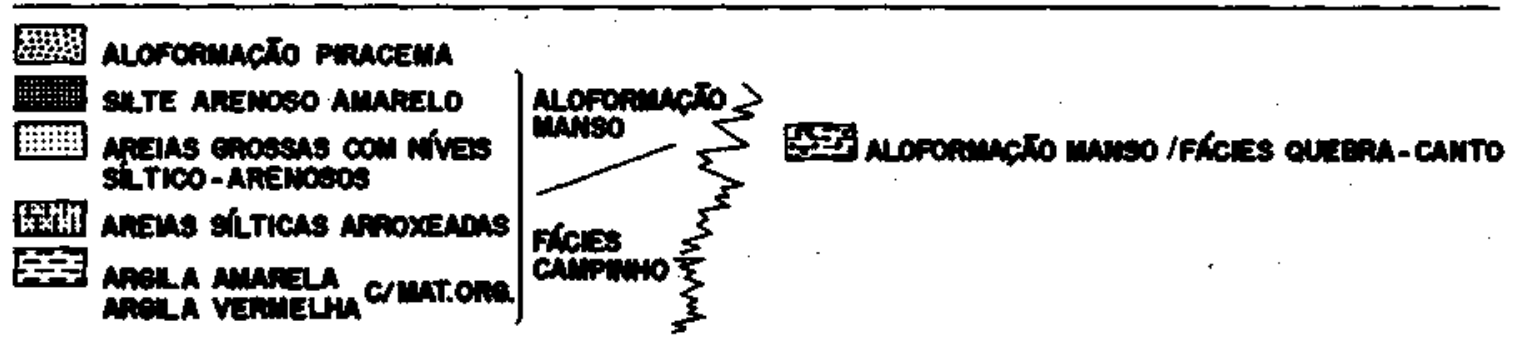

Figura 4 - Seção Turvo, localizada na estrada Bananal (SP) - Rialto (RJ), a $15 \mathrm{~km}$ de Bananal. Reproduz, a interdigitação entre depósitos aluviais e alúvio-coluviais no domínio de articulação do eixo de uma sub-bacia de drenagem entulhada com o nível de terraço superior - TI (Moura \& Mello 1989)

Figure 4 - Turvo Section, localized on Bananal (SP) - Rialto (RJ) road, $15 \mathrm{~km}$ from Bananal. The figure shows alluvial deposits intetfingering with alluvial-colluvial ones in the articulation zone of "drowned" sub-basin axis with superior terrace level - T1 (Moura \& Mello 1989)

permaneceram em agradação, o recuo das encostas foi incipiente, traduzindo-se em formas semicirculares de gradiente suave na concavidade basal. Naquelas onde a degradação das encostas foi mais acentuada, é possível observar o reafeiçoamento da topografia pelas unidades coluviais mais recentes.

Os anfiteatros com hollow côncavo suspenso podem resultar de fenômenos erosivos controlados por níveis de base locais da própria encosta (movimentos de massa e/ou voçorocas). Nesse caso, configuram-se como cabeceiras de forma cônica, embutidas no segmento superior das encostas, sendo denominados anfiteatros com hollow côncavo suspenso embutido (HCSe) (Foto 5)

Os anfiteatros e sub-bacias de drenagem com hollow côncavo-plano podem apresentar-se parcialmente reafeiçoados, caracterizando-se pela articulação ligeiramente suavizada das encostas laterais e frontal com o fundo plano. Este subtipo é denominado anfiteatro com hollow côncavo-plano reaf eiçoado (HCPr) (Foto 6).

A distribuição espacial dos tipos de cabeceira de drenagem apresentados pode ser obtida por meio do mapeamento das feições geomórficas associadas à estrutura de subsuperfície, em escala apropriada (Fig. 8). Os anfiteatros e sub-bacias de drenagem com hollow côncavo-plano (HCP) estão, atualmente, em sua maioria, em processo de reierarquização hidrográfica pela retomada erosiva por voçorocas (Peixoto et al 1989).

CONSIDERAÇÕES FINAIS Os estudos sobre expansão e desenvolvimento de fluxos canalizados, bem como quaisquer outros processos que impliquem na remoção de material das cabeceiras de drenagem não-canalizadas, somente podem fornecer subsídios para a modelagem matemática sobre o seu desenvolvimento morfológico quando aliados a um certo grau de informações sobre a evolução do sistema em escala de tempo geológico, por sua vez, só obtidas por meio da análise dos registros estratigráficos. A Estratigrafia surge como instrumento imprescindível à interpretação das formas (Johnson 1982), constituindo o elo de Ugação entre processos passados e presentes e possibilitando a previsão dos processos futuros dentro do contexto evolutivo do modelado.

Inserida nessa perspectiva, a morfologia das vertentes é a resultante atual de sucessivos reafeicoamentos, que poderão apagar ou não os vestígios de episódios anteriores. A seqüência estratigráfica, mesmo quando localmente incompleto, poderá fornecer indícios materiais da complexidade evolutiva.

A integração de argumentos de cunho geomorfológico e estratigráfico sobre a dinâmica quaternária permitiu que os conhecimentos sobre a evolução das encostas nos trópicos úmidos fosse aprimorado. As descontinuidades na geometria de superfície e subsuperfície dos complexos de rampa, em seus segmentos transversais e longitudinais, podem ser indicadores de fases diferenciadas da evolução do anfiteatro. Os anfiteatros constituem importantes unidades funcionais para a análise geomorfológica.

No caso estudado, as feições de hollow côncavo, associadas à dinâmica de remoção e retrabalhamento dos regolitos no domínio das encostas, representam grande parte das reentrâncias que configuram em planta a morfologia convexo-côncava das colinas do Planalto SE do Brasil.

Os HCP, associados a fenômenos de instabilidade ambiental durante o Holoceno (paleovoçoroças), desenvolvem-se nos eixos principais das sub-bacias de drenagem onde a coalescência de anfiteatros sugere um maior grau de hierarquização. Podem estar relacionados a eixos secundários (pequenas cabeceiras de drenagem) e, neste caso, resultam de deslocamen- 

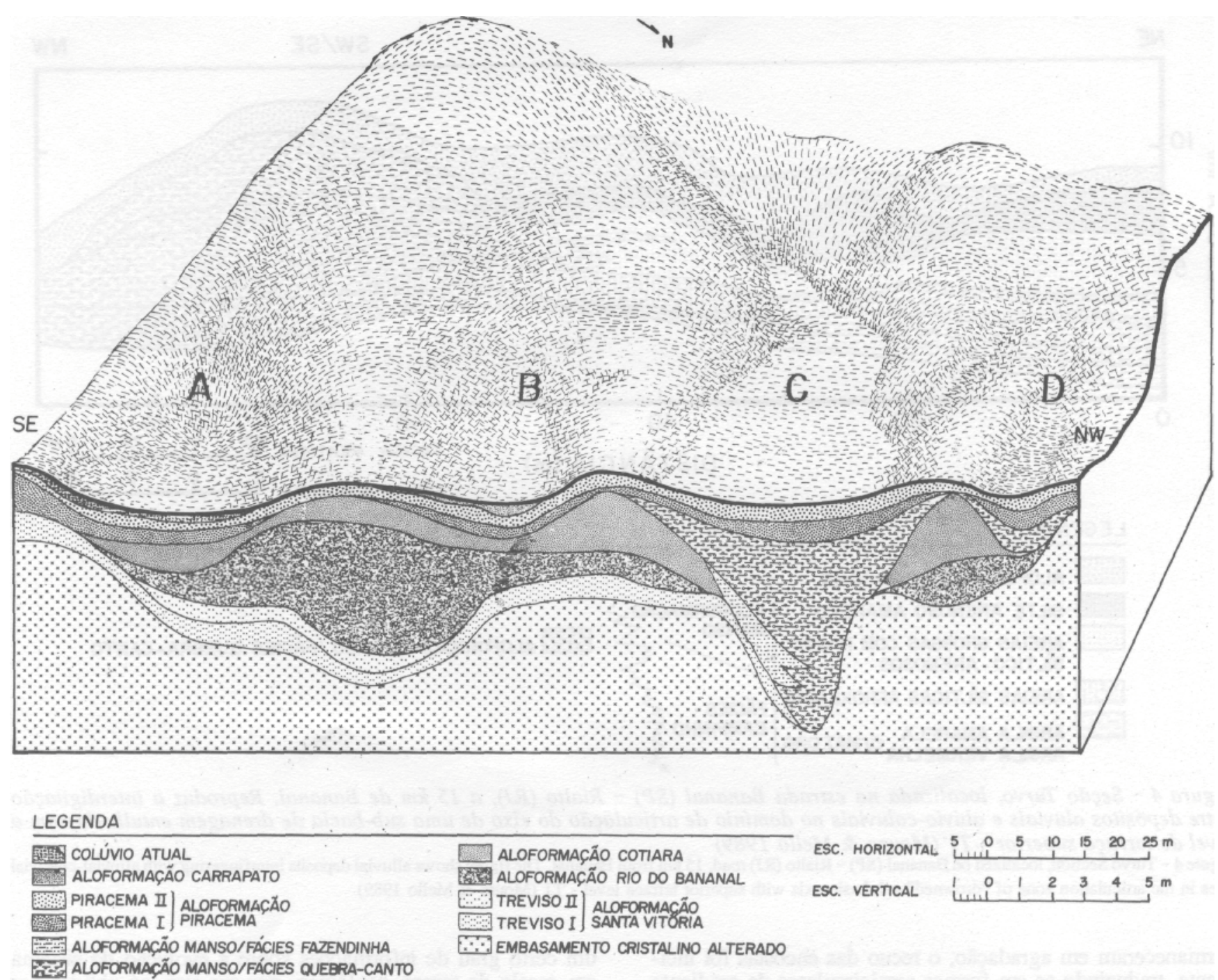

Figura 5 - Bloco - diagrama com a reconstituição estratigráfica da Seção Bela Vista, situada na estrada Bananal (SP) - Rialto (RI), a $13 \mathrm{~km}$ de Bananal. A e B constituem hollows côncavos, demonstrando em sua estrutura subsuperficial sucessivos retrabalhamentos de unidades coluviais; $C$ e D correspondem a hollows côncavos-planos, destacando-se em subsuperficie a presença de depósitos alúvio-coluviais. D apresenta-se parcialmente reafeiçoado

Figure 5 - Diagram-block with Bela Vista Section stratigraphy, situated on Bananal (SP) - Rialto (RJ) road, $13 \mathrm{~km}$ from Bananal. A and B are concave hollows, showing in its subsuperficial structure successive colluvial units reworking; $\mathrm{C}$ and $\mathrm{D}$ correspond to concave-plane hollows, showing in its subsuperficial structure the alluvial-colluvial deposits. Note that $\mathrm{D}$ is partialy reshaped

tos das linhas de fluxo que originaram inversões na topografia dos complexos de rampa (Moura et al. inédito), Na região de Bananal (SP/RJ), a distribuição dos HCP acompanha nitidamente alinhamentos preferenciais, semelhante ao padrão da drenagem, sugerindo condicionamento estrutural.

As feições geométricas e a natureza dos materiais deposicionais devem ser consideradas como o resultado da atuação dos processos ao longo do tempo e como fator de distribuição espacial dos processos atuais (Meis \& Moura 1984). Oliveira $\&$ Meis (1985), relacionando as formas erosivas à geometria das encostas, verificaram que $66 \%$ das voçorocas mapeadas em uma área situada em Bananal (SP) e municípios vizinhos concentravam-se nos domínios côncavos em planta e perfil. Estudos realizados por Peixoto et al (1989), para a região de Bananal (SP/RJ), indicam que as cabeceiras de drenagem com hollow côncavo-plano (HCP) constituem os ambientes onde a retomada erosiva é predominante: os HCP apresentam $60 \%$ de casos de erosão linear acelerada, enquanto os HCS apresentam $20 \%$ e os HCA, $12 \%$. Trata-se de um mecanismo em que a retomada erosiva atual tem direcionamento preferencial para as antigas linhas de drenagem, através de voçorocas remontantes a partir do rompimento de pacotes aluviais e alúvio-coluviais, sugerindo o controle direto dos níveis de base das calhas fluviais, ou seja, uma reierarquização do sistema de drenagem. Os padrões evolutivos de anfiteatros - extensivos às subbacias de drenagem entulhadas - apreendidos a partir da tipologia apresentada, podem subsidiar a compreensão da distribuição diferencial dos solos, os estudos hidrológicos de encostas e fluxos canalizados e, conseqüentemente, a elucidação do desencadeamento dos fenômenos erosivos atuais. Da mesma forma, é possível sua aplicabilidade nas questões referentes ao planejamento do uso do solo.

Agradecimentos A realização deste estudo recebeu o apoio financeiro da FINEP, CNPq, FAPERJ e CEPG/UFRJ. Desejamos agradecer aos demais integrantes do Grupo de Estudos em Geomorfologia e Estratigrafía do Quaternário GEOQUAT (IGEO/UFRJ), pela colaboração em todas as etapas do desenvolvimento do trabalho e ao prof. Josué Camargo Mendes pela revisão crítica do artigo. 


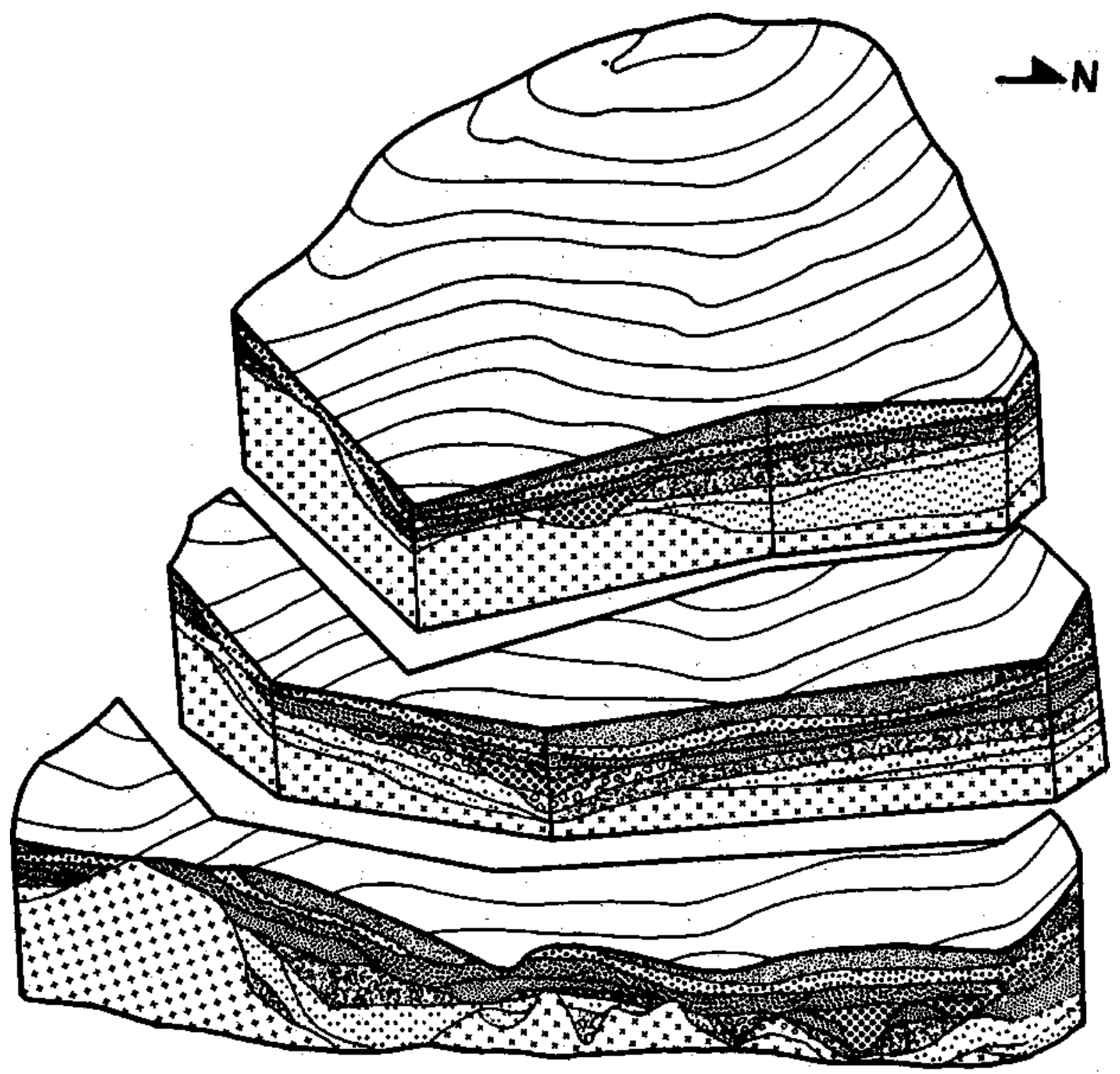

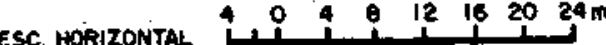

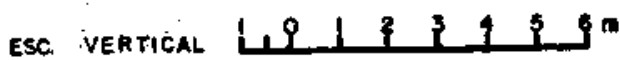

LEGENDA:

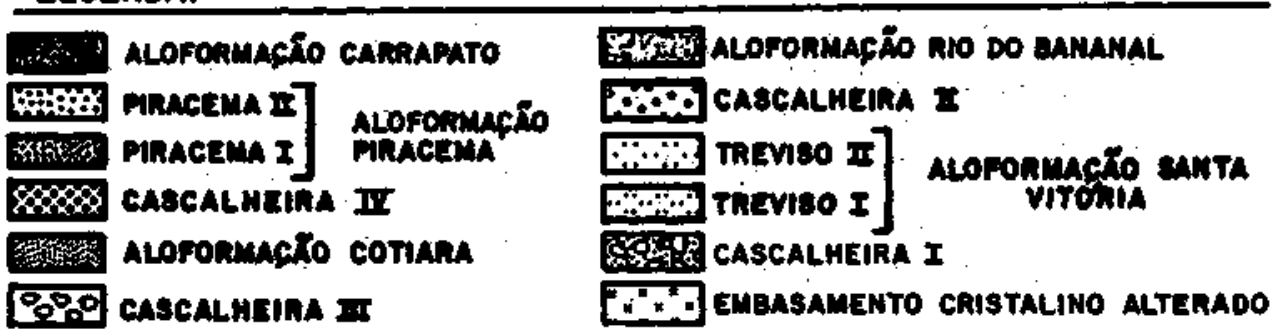

Figura 6 - Bloco-diagrama com a representação tridimensional da estratigrafia do anfiteatro Treviso, localizado na estrada Bananal (SP) - Rialto (RJ), a 4,5 km de Bananal As várias fases de erosão linear acelerada, documentadas pelas cascalheiras I, II, III e IV, estão mascaradas pelas fases de coluviação posteriores

Figure 6 - Diagram-block with tridimensional representation of Treviso amphitheater stratigraphy, situated on Bananal (SP) - Rialto (RJ) road, $4.5 \mathrm{~km}$ from Bananal. The various phases of accelerated linear etoson, documented by L, H, HI and IV gravel layers are masked by posterior colluviation phases 


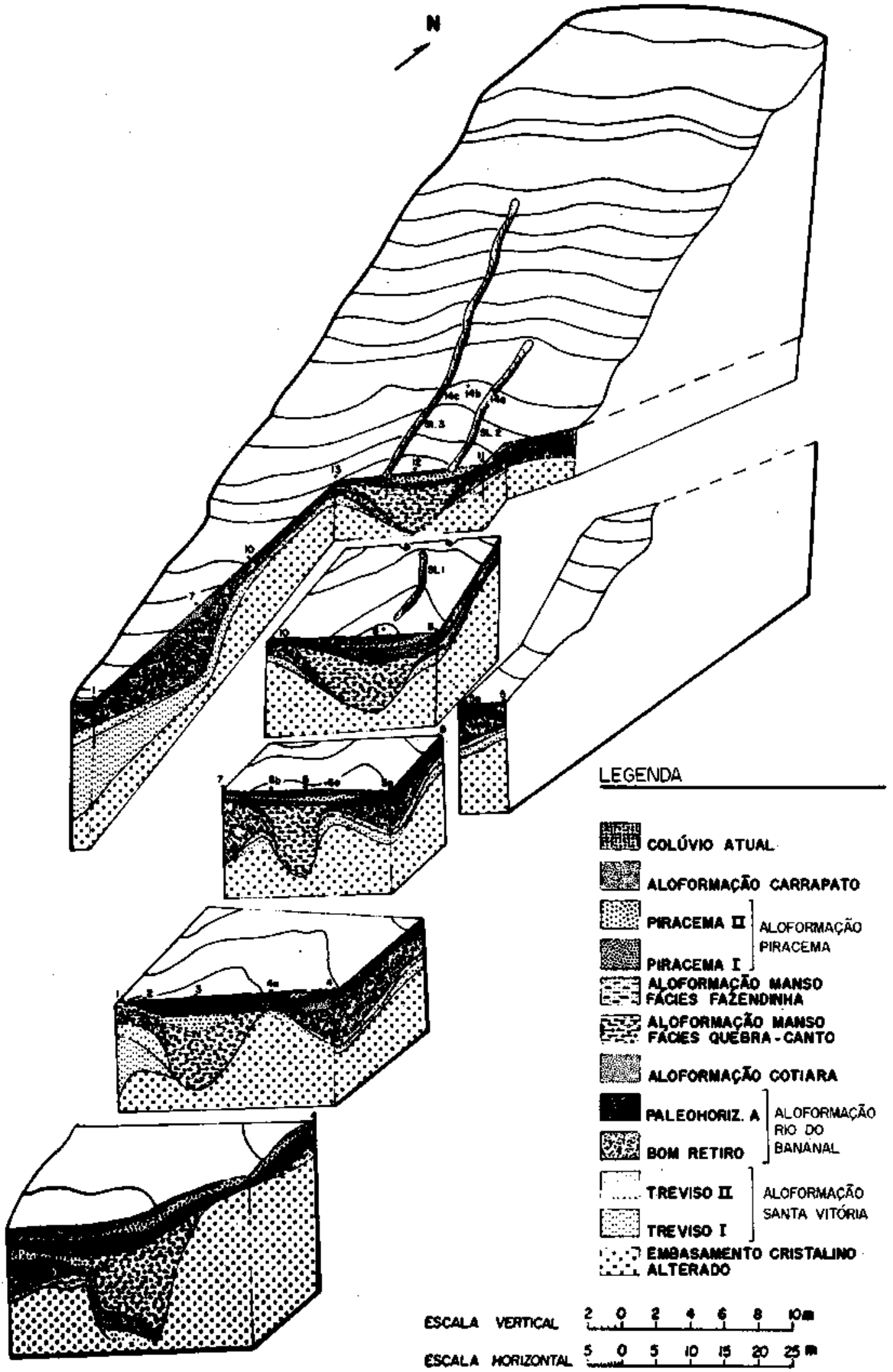

Figura 7 - Bloco-diagrama com a representação tridimensional da estratigrafia do anfiteatro Bom Retiro H, situado na estrada Bananal (SP) - Rialto (RJ), a $1,5 \mathrm{~km}$ de Bananal $O$ paleocanal erosivo está preenchido por materiais alúvio-coluviais. Os números assinalados representam pontos de sondagem; SL1, SL2 e SL3 identificam voçorocas descontínuas atuais Figure 7 - Diagram-block with tridimensional representation of Bom Retiro H amphitheater stratigraphy, situated on Bananal(SP) - Rialto (RJ) road 1.5 km from Bananal. Observe the erosive paleochannel filed by alluvial-colluvial materials. The numbers represent drillholes; SLl, SL2 and SL3 identify actual discontinuous gullies 

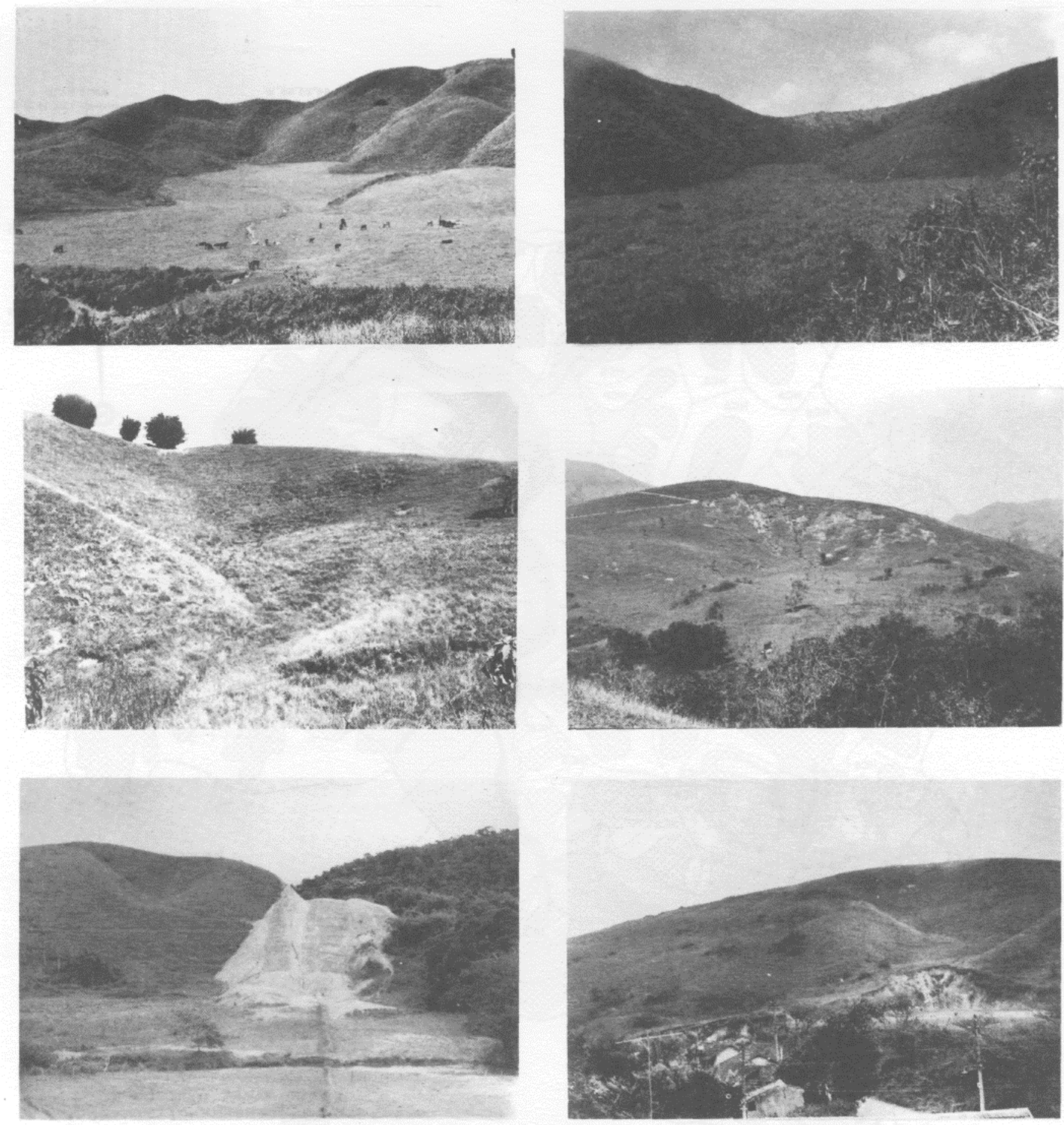

Prancha l-Foto 1 - Sub-bacia de drenagem entulhada, associada ao eixo principal da drenagem, localizada a $2 \mathrm{~km}$ de Bananal (SP), na estrada de acesso à Fazenda Santa Apolônia; Foto 2-Anfiteatro com hollow côncavo-plano (HCP) relacionado ao eixo secundário da drenagem, localizado a $8 \mathrm{~km}$ de Bananal (SP), na estrada de acesso à Fazenda Bela Vista; Foto 3 Anfiteatro com hollow côncavo articulado (HCA), localizado na estrada Bananal-Arapei (SP-066), a 2 km de Bananal (SP);

Foto 4 - Anfiteatro com hollow côncavo suspenso (HCS), localizado na estrada Bananal - Serra da Bocaina (SP-247), a 6 $\mathrm{km}$ de Bananal. O processo de erosão está acelerado, desarticulado em relação á drenagem; Foto 5 - Anfiteatro com hollow côncavo suspenso embutido (HCSe), localizado na estrada Bananal (SP) - Rialto (RJ), a 14 km de Bananal; Foto 6-Anfiteatro com hollow côncavo-plano reafeiçoado (HCPr), situado na localidade conhecida como $\mathrm{km} 4$, distrito de Cotiara, Barra Mansa $(R J)$

Plate 1 - Photo 1 - "Drowned" sub-basin, associated to main drainage axis; situated 2 km from Bananal (SP), on the road to Santa Apolônia Farm; Photo 2 - Amphitheaterlike head-water with concave-plane hollow (HCP) associated to secondary drainage axis, located $8 \mathrm{~km}$ from Bananal (SP), on the road to Bela Vista Farm; Photo 3 - Amphitheaterlike head-water with articulate concave hollow (HCA), situated on Bananal - Arapeí road (SP - 066), 2 km from Bananal (SP); Photo 4 - Amphiteaterlike head-water with suspended concave hollow (HCS), located on Bananal - Serra da Bocaina road (SP-247), 6km from Bananal. Observe accelerated erosion process, disarticulate from the channel; Photo 5 - Amphitheaterlike head-water with inlaid suspended concave hollow (HCSe), situated on Bananal (SP) - Rialto (RJ) road, 14 km from Bananal; Photo 6 - Amphitheaterlike head-water with reshaped concave-plane hollow (HCPr), situated in km 4 locality, Cotiara district, Barra Mansa (RJ) 


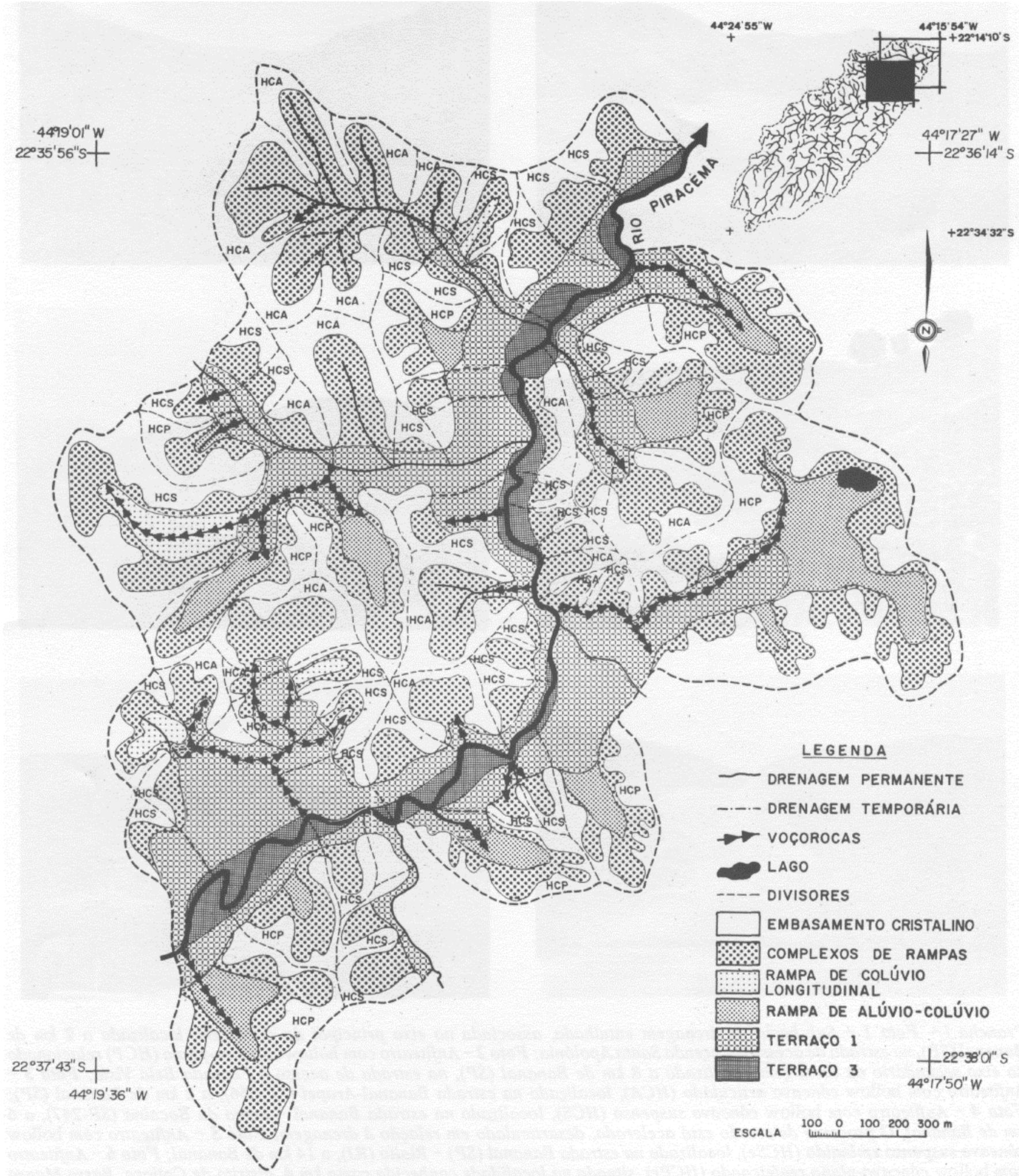

Figura 8 - Mapeamento geomorfológico de um segmento do médio-baixo curso da bacia do Rio Piracema, região de Bananal $(S P / R J)$. Os anfiteatros e sub-bacias de drenagem entulhadas foram delimitados e classificados segundo a tipologia proposta (Peixoto et aL 1989)

Figure 8 - Geomorphologic map of a segment of middle Piracema river valley, Bananal region (SP/RJ). The amphitheaterlike head-waters and the "drowned" sub-basins were delimitated and classified according to the proposed typology (Peixoto et al. 1989) 


\section{REFERÊNCIAS BIBLIOGRÁFICAS}

AHRNNET, F. (ed.) 1985. Soils and Geomorphology. New York, Wiley \& Sons. $347 \mathrm{p}$

BIERKLAND, P.M. 1974. Pedology, Weathering and Geomorphological Research. New York, Oxford Uoiv. Press. 285 p.

BIGARELLA, J.J. \& MOUSINHO, M.R. 1965. Considerações a respeito dos terraços fluviais, rampas de colúvio e várzeas. Bol. Paran. Geogr., 16/17:153-197.

BOARDMAN, J. (ed.) 1985. Soils and Quaternary Landscape Evolution. New York, Wiley \& Sons. 319 p.

CARSON, MA. \& KIRKBY, M.J. 1972. Hillslope Form and Process. London, Cambridge Univ. Press. 475 p.

CHORLEY, R.J. 1964. The model position and anomalous chanter of slope studies in geomorphological research. Geogr. J., 130:503-506.

DIETRICH, WE.; WILSON, C.J.; RENEAU, S.L. 1986. Hollows, landslides and colluvium in soil-mantled landscapes. In: ABRAHAMS, A.D. ed. Hillslope Processes. Boston, Allen \& Unwin. p. 361-388.

FANCRAN, A. \& AJAEGBU, H. 1971. Landslopes and form sizes in a part of Jos plateau. Nig. Geogr. J.. 14(2): 199-207.

FANIRAN, A. \& JEJE, L.K. 1983. Humid Tropical Geomorphology. New York, Longman. $414 \mathrm{p}$.

GERRARD, A.J.W. 1981. Soils and Land/arms, an Integration of Geomorphology and Pedology. London, Allen \& Unwin. 162p.

HACK, J.T. \& GOODLETT, J.C. 1960. Geomorphology and forest ecology of a mountain region in the central Appalachians. Denver, U.S. Geol. Surv. 66 p. (Prof. Paper 347).

HUGGET, R.J. 1975. Soil Landscape System: a model of soil genesis. Geoderma, 75:1-22.

JEJE, L.K. 1976. Slope profile from a humid tropical environment, the example of Opa basin in If e area. Bull. Ghana Geog. Assn., 18:52-67.

JOHNSON, W.H. 1982. Interrelationships among geomorphic interpretations of the stratigraphic record, processes, geomorphology and geomorphic models. In: THORN, C.E. ed. Space and Time in Geomorphology. London, The Binghampton Symposia in Geomorphology. p. 219-241

KIRKBY, M.J. 1971. Hillslope process-response models based on the continuity equation. London, Inst Br. Geogr., 3:15-30. (Spec. Public.).

KIRKBY, MJ. \& CHORLEY, RJ. 1967. Through flow, overland flow and erosion. Local, Bull. Int. Assoc. Sci. Hydrol, 12:5-21.

MACHADO, M.B. \& MOURA, J.R.S. 1982. A geomorfologia e a sedimentação quaternária no médio Vale do Rio Casca, MG. Ia: CONOR. BRAS. GEOL., 32. Salvador, 1982. Anais... Salvador, SBG. v. 4 ; p. 1433-1441.

MEIS, M.R.M. 1977. As unidades morfoestratigráfícas neoquatemárias do médio vale do Rio Doce. An. Acad. bras. Cienc., 49:443-459.

MEIS, M.R.M. \& MACHADO, M.B. 1978. A morfologia de rampas e terracos no Planalto Sudeste do Brasil. Finisterra, 13(26):201-218.

MEIS, M.R.M. \& MONTEIRO, A.M.F. 1979. Upper Quaternary "Rampas": Doce River Valley, SE Brazilian Plateau. Z. Geomorphol, 23(2): 132-151.

MEIS, M.R.M. \& MOURA, J.R.S. 1984. Upper Quaternary Sedimentation and Hillslope Evolution: SE Brazilian Palteau. Am. J. Sci., 284:241-254.
MEIS, MJLM.: COELHO NETTO, A.L.; MOURA, JJLS. 1985. As descontinuidades nas formações coluviais como condicionantes dos processos hidrológicos e da erosão acelerada. In: SIMP. NACIONAL DE CONTROLE A EROSÃO, 3. Maringá, 1985. Anais... Maringá, ABGE. p.179-189.

MOURA, JJLS. \& MEIS, MJLM. 1980. Litoestratigrafia preliminar para os depósitos de encosta do Quaternário Superior do Planalto SE do Brasil (MG-RJ). Rev. Bras. Geoc., 10(4):258-267.

MOURA, J.R.S. \& MEIS, MJLM. 1986. Contribuição à estratigrafia do Quaternário Superior no médio Vale do Rio Paraíba do Sul - Bananal, SP. An. Acad. bras. Ciênc., 58:89-102.

MOURA, JJLS. \& MELLO, CL. 1989. Aloformacão Manso: um episódio de instabilidade ambiental nó Holoceno do Planalto SE do Brasil. In: CONOR. BRAS. PALEONTOLOGIA, 11. Curitiba, 1989. Anais... Curitiba, SBP. v. 2, p. 1073-1083.

OFOMATA, G.EJC. 1973. Aspects of the geomorphology of the Nsukka-Okigwe cuesta, East Central State of Nigeria. BulL Inst.fondam. d'Afrique Noire, 35-A(3);489-501.

OLIVEIRA, MA.T. \& MEIS, MJLM. 1985. Relações entre geometria do relevo e formas de erosão linear acelerada (Bananal, SP). Geociências, 4:87-99.

PEIXOTO, M.N.O.; MOURA, J.R.S.; SILVA, T.M. 1989. Morfometria de cabeceiras de drenagem em anfiteatros e a retomada erosiva por voçorocamento - Bananal (SP). In: SIMP. GEOGR. FÍS. APLICADA, 3. Nova Friburgo. 1989. Anais... Rio de Janeiro, IGEO/UFRJ. p. $149-174$.

RUHE, R.V. 1975. Geomorphology - Geomorphic Process and Surficial Geology. Boston, Houghton Mifflin Company. 254 p.

SWAN, S.B. St.C. 1970. Relationship between regolith, lithology and slope in a humid tropical region: Johor, Malaya. Trans. Inst. Br. Geogr., $51: 189-200$.

TROEH, F.H. 1965. Landform equations fitted to contour maps. Am. J. Sci., 263:616-627.

TSUKAMOTO, Y. OHTA, T.; \& NOGUCHJ, H. 1982. Hydrological and geomorphological studies of debris slides on forested hillslopes in Japan. In: WALLING, D.E. ed. Recent Developments in the Exploration and Prediction of Erosion and Sediment Yield. Oxford, Int Asssoc. Hydrol. Sci., v. 137. p. 89-98.

YOUNG, A. 1970. Slope form in pan of the Mato Grosso, Brasil. Geogr. $J_{.,}, 136: 383-392$.

YOUNG, A. 1972. Slopes. London, Longman Group. $288 \mathrm{p}$.

WIGWE, G.A. 1966. Drainage Composition and Galley Forms in Parts of Northern and Western Nigeria. Idaban. 462p. (PhD thesis, University of Idaban).

MANUSCRTTO A635 Recebldo em 11 de derembro de 1939

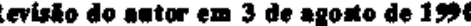
Rerletio ceetith eim 9 de agodo de 199 